SWAT/94-95/57 UM-P-94/122 QMW-PH-94-39 hep-th/9501078

\title{
Restricted Quantum Theory of Affine Toda Solitons
}

\author{
B. Spence \\ School of Physics \\ University of Melbourne \\ Parkville 3052 Australia \\ E-mail:B.Spence@qmw.ac.uk \\ Jonathan Underwood \\ Physics Department \\ University College of Swansea \\ Swansea SA8 2PP UK \\ E-mail:J.Underwood@swan.ac . uk
}

\begin{abstract}
We quantise the reduced theory obtained by substituting the soliton solutions of affine Toda theory into its symplectic form. The semi-classical S-matrix is found to involve the classical Euler dilogarithm function.
\end{abstract}

\footnotetext{
${ }^{1}$ Current Address: Physics Department, Queen Mary \& Westfield College, Mile End Road London E1 4NS UK
} 


\section{Introduction}

The affine Toda field theories associated to an affine Kac-Moody algebra $\hat{\mathrm{g}}$ have received a great deal of attention in recent years. The classical equation of motion which describes them is

$$
\square^{2} \phi+\frac{2 \mu^{2}}{\beta} \sum_{i=0}^{r} m_{i} H_{i} e^{\beta \alpha_{i}(\phi)}=0,
$$

where $\phi$ is the dynamical field, taking values in the Cartan subalgebra of $\hat{\mathbf{g}}$ (for whom Chevalley generators are the $H_{i}$ and the Coxeter number is $\left.h\right), \mu$ and $\beta$ are real parameters, and $\alpha_{i}$ are the simple roots of $\hat{\mathbf{g}}$. Taking $\beta$ to be real we obtain a model with a particle spectrum whose properties are algebraic in origin (see, for example, refs. [1, 2]), and whose S-matrix is exactly known [3, 2, 4, 5, 6, 0, 8, 9, 10].

If the coupling constant $\beta$ is pure imaginary then the model possesses a series of vacuum solutions. We expect soliton solutions to be those of minimal energy which interpolate these vacua, the difference between the final and the initial vacua being known as the topological charge of the soliton. The seminal calculation was made by Hollowood [11] for the $\hat{A}_{r}$ models using Hirota's method. This technique was later developed to include the rest of the theories 12, 13. A new method, involving the use of the general solution discovered by Leznov and Saveliev (see e.g. [14]), has recently proved to be very useful in extracting particular properties of the solitons [15]. This method can be motivated by consideration of the form of the canonical energy-momentum tensor

$$
T_{\mu \nu}=\left(\partial_{\mu} \phi, \partial_{\nu} \phi\right)-\frac{g_{\mu \nu}}{2}\left(\partial_{\alpha} \phi, \partial^{\alpha} \phi\right)-\frac{2 g_{\mu \nu}}{\beta^{2}} \sum_{k=0}^{r} n_{k}\left(e^{\beta \alpha_{k}(\phi)}-1\right) .
$$

Using arguments given in ref. [15] it can be shown that $T_{\mu \nu}$ can be split into a sum of two parts, $T_{\mu \nu}=C_{\mu \nu}+\Theta_{\mu \nu}$, where $\Theta_{\mu \nu}$ is traceless and $C_{\mu \nu}$ is a total derivative. Due to the topological nature of solitons, their properties, in particular their energies and momenta, depend only on their behaviour at infinity, and hence we might expect $\Theta_{\mu \nu}$ to vanish for soliton solutions. Further work 115, 16] shows that $\Theta_{\mu \nu}$ can be written purely in terms of the chiral fields which are the parameters of the Leznov-Saveliev solution, so it is plausible that the soliton solutions arise if these fields vanish.

In order to find the $\mathrm{N}$-soliton solutions a further ansatz for the form of a constant of integration appearing in the general solution must be made 17; the N-soliton specialised solution turns out to be

$$
e^{-\beta \phi_{k}}=\left\langle\Lambda_{k}\left|\exp \left(W_{1} \hat{F}^{i_{1}}\left(z_{1}\right)\right) \ldots \exp \left(W_{N} \hat{F}^{i_{N}}\left(z_{N}\right)\right)\right| \Lambda_{k}\right\rangle
$$


For a detailed explanation of the quantities appearing in the formula the reader should consult ref. [17]; we will need only the following details. The parameters $i_{j}$ are integers lying between 1 and $r$ which describe the species of the $\mathrm{N}$ solitons. $z_{j}$ are complex parameters related to the soliton rapidities $\rho_{j}$ in the following way:

$$
z_{j}=\theta_{j} e^{-\rho_{j}} \frac{\left|q_{i_{j}}^{+}\right|}{q_{i_{j}}^{+}},
$$

where $\theta_{j}= \pm 1$, and the $q_{i_{j}}$ are structure constants of $\hat{\mathrm{g}}$ which can be calculated from the following formula of ref. [1]

$$
q_{p}^{+} \equiv \gamma_{p} \cdot q(1)=2 \imath x_{p}(1) e^{-\delta_{p B} \imath \nu \pi / h}
$$

$(1 \equiv \sqrt{-1})$. The functions $W_{j}$ encode the space-time dependence of the solution, and are given by the formula

$$
W_{j}=Q_{j} \exp \left\{\sqrt{2} \mu \theta_{j}\left|q_{i_{j}}^{+}\right|\left(t \sinh \rho_{j}-x \cosh \rho_{j}\right)\right\}
$$

The first major use to which this formalism was put was a calculation of the masses of single solitons of species $p$. These turned out to be [15]:

$$
M_{p}=-\frac{4 \sqrt{2} h}{\beta^{2} \gamma_{p}^{2}} \mu\left|q_{p}^{+}\right|
$$

An interesting fact which is not yet fully understood is that these masses are in a certain sense dual to those of the particles.

In this letter we will use further features of the solution (1.3) to calculate the Poisson brackets on the N-soliton phase space and will perform a canonical quantisation to extract the S-matrix.

\section{Poisson Brackets}

The classical phase space of the affine Toda theories has been discussed recently in ref. [18]. The symplectic form $\Omega$ is calculated by integrating the symplectic current over all space at some time, and is simply

$$
\Omega=\int d x\left(\delta \phi, \delta \partial_{t} \phi\right)
$$

where $($, ) denotes the usual Killing form on the algebra $\hat{\mathbf{g}}$.

We wish to investigate the phase space of the soliton solutions. To do this, we insert the soliton solutions into the symplectic form $\Omega$. First let us calculate the symplectic form for a one-soliton solution. We will need to use two important properties of this solution, but our method will mean that we do not need the explicit 
form of the solution itself. This is a typical feature of calculations concerning solitons in affine Toda theory. The first feature we need is that the soliton is a relativistic object, and so the Poincare algebra must be realised on the phase space. What this means for the solution is that the field $\phi$ must appear only as a function of $u$, where

$$
u=t \sinh \rho-\left(x-x_{0}\right) \cosh \rho .
$$

The rapidity $\rho$ and centre of mass $x_{0}$ are the real parameters of the soliton solution. The relationship of these parameters to those in the algebraic ansatz for the soliton solution, as well as the explicit dependence of $\phi$ upon $u$, can be determined from the formulæ of [15], [17, but we shall not need these yet. Using the antisymmetry properties of the wedge product we obtain

$$
\begin{aligned}
\Omega & =\int d x\left(\frac{d \phi}{d u}, \frac{d \phi}{d u}\right) \cosh \rho \delta x_{0} \wedge \delta(\sinh \rho), \\
& =-\int d x\left(\partial_{t} \phi, \partial_{x} \phi\right) \frac{\delta x_{0} \wedge \delta(\sinh \rho)}{\sinh \rho}, \\
& =-\int T_{t x} \frac{\delta x_{0} \wedge \delta(\sinh \rho)}{\sinh \rho},
\end{aligned}
$$

where the last step is performed using the expression for the energy-momentum tensor (1.2). Finally, substituting $P=-\int d x T_{01}=M \sinh \rho$ into (2.3), we obtain

$$
\Omega=\delta \xi \wedge \delta \rho,
$$

where $\xi=M x_{0} \cosh \rho$ is the canonical variable conjugate to $\rho$. This is of course the result we expect.

In order to evaluate the symplectic form in the more general case of $N$ solitons we consider the form of the solution as $t \rightarrow \pm \infty$. In the generic situation the solitons will be well separated and since $\Omega$ is a local expression we can just add up the contributions from each of the solitons in turn. Thus we find

$$
\Omega=\sum_{i=1}^{N} \delta \xi_{i}^{\text {out }} \wedge \delta \rho_{i}^{\text {out }} \text { in }
$$

We now need to relate these variables to those which parameterise the solution.

Let us consider the form of the solution (1.3) as $x$ increases from $-\infty$. The first significant departure from the vacuum will occur with the soliton of greatest rapidity, $\rho_{N}$. We know from ref. [17] that the greatest non-vanishing power of $\hat{F}^{i_{N}}$ within a representation of level $x$ is $x$. 2 This in turn means that the solution for the component field $\phi_{k}$ will be the logarithm of a polynomial of degree $m_{k}$ in $W_{N}$ defined by equation (1.6). As we move through this soliton $W_{N}$ becomes much

\footnotetext{
${ }^{2}$ We are only considering the theories where $\hat{\mathbf{g}}$ is simply-laced from now on.
} 
greater than 1 and so we can ignore all but the highest power in the polynomial as far as calculating the form of the solution for greater $x$ is concerned. This term of course multiplies an algebraic factor $\hat{F}^{i_{N}}\left(z_{N}\right)$. Normal ordering the vertex operator expression [19], 17] for this yields

$$
\begin{array}{r}
e^{-\beta \phi_{k}}=\left\langle\Lambda_{k}\left|F^{i_{N}}\left(z_{N}\right)\right| \Lambda_{k}\right\rangle W_{N}^{m_{k}}\left\langle\Lambda_{k}\right| \exp \left(X_{i_{1}, i_{N}}\left(z_{1}, z_{N}\right) W_{1} \hat{F}^{i_{1}}\left(z_{1}\right)\right) \ldots \\
\ldots \exp \left(X_{N-1, N}\left(z_{N-1}, z_{N}\right) W_{N-1} \hat{F}^{i_{N-1}}\left(z_{N-1}\right)\right)\left|\Lambda_{k}\right\rangle,
\end{array}
$$

where

$$
X_{i, j}\left(z_{1}, z_{2}\right)=\prod_{n=0}^{h-1}\left(1-e^{-2 \pi i n / h} \frac{z_{2}}{z_{1}}\right)^{w^{n}\left(\gamma_{i}\right) \cdot \gamma_{j}} .
$$

The roots $\gamma_{i}$ are the simple roots $\alpha_{i}$ up to a sign [17], and $w$ is the Coxeter element of the Weyl group. Thus, aside from a constant shift in the field $\phi$, the only effect is to change each of the $Q_{j}$ for $j<N$ by a factor $X_{i_{j}, i_{N}}\left(z_{j}, z_{N}\right)$. Extending this argument as we move through each of the solitons in turn leads us to conclude that as $t \rightarrow-\infty$ the field $\phi$ becomes a sum of one-soliton solutions but with new parameters

$$
Q_{j}^{\mathrm{in}}=Q_{j} \prod_{p>j} X_{j, p}\left(z_{j}, z_{p}\right)
$$

The rapidities remain unchanged under the transformation to 'in' variables. Now all we need to do is relate the variables $Q_{j}$ to the $\xi_{j}$. Comparing expressions (1.6) and (2.2) we find that up to an irrelevant constant

$$
x_{0}=\frac{\theta \ln Q}{\sqrt{2} \mu\left|q_{i}^{+}\right| \cosh \rho} .
$$

Using the mass formula equation (1.7) we obtain

$$
\xi=\frac{2 h \theta \ln Q}{|\beta|^{2}}
$$

and so

$$
\begin{aligned}
\xi_{j}^{\text {in }} & =\xi_{j}+\frac{2 h}{|\beta|^{2}} \sum_{p>j} \ln X_{j, p}\left(z_{j}, z_{p}\right), \\
\rho_{j}^{\text {in }} & =\rho_{j} .
\end{aligned}
$$

Similar arguments yield the following relationships for the out variables

$$
\begin{aligned}
& \xi_{j}^{\text {out }}=\xi_{j}+\frac{2 h}{|\beta|^{2}} \sum_{p<j} \ln X_{p, j}\left(z_{p}, z_{j}\right), \\
& \rho_{j}^{\text {out }}=\rho_{j} .
\end{aligned}
$$


Since the symplectic form $\Omega$ is trivial to invert in terms of terms of either 'in' or 'out' variables the appropriate Poisson brackets for the natural variables follow straightforwardly, and can be seen to be consistent if we note that $X\left(z_{j}, z_{p}\right)$ depends only on the rapidity difference $\rho_{j}-\rho_{p}$.

\section{Scattering Matrix}

Having found canonical coordinates on the classical phase space we can now quantise the theory in a straightforward manner, simply by replacing the canonical Poisson bracket with the canonical commutators

$$
\left[\xi^{\text {in }}, \rho^{\text {in }}\right]=\left[\xi^{\text {out }}, \rho^{\text {out }}\right]=\imath \hbar .
$$

We can then attempt to discover the unitary transformation (analogue of the canonical transformation) which will be the S-matrix for the reduced theory. From equations (2.11) and (2.12) we conclude that

$$
\begin{aligned}
& \xi_{j}^{\text {out }}=\xi_{j}^{\text {in }}+\frac{2 h}{|\beta|^{2}} \sum_{p<j}\left(\ln X_{p, j}\left(z_{p}, z_{j}\right)-\sum_{p>j} \ln X_{j, p}\left(z_{j}, z_{p}\right)\right) \\
& \rho_{j}^{\text {out }}=\rho_{j}^{\text {in }}=\rho_{j} .
\end{aligned}
$$

We can see from these equations that the $\mathrm{S}$ matrix $S$ is purely a function of the rapidity differences, and satisfies the equation

$$
\frac{\partial(\log S)}{\partial \rho_{j}}=\frac{-2 h \mathrm{1}}{|\beta|^{2} \hbar}\left(\sum_{p<j} \ln X_{p, j}\left(\rho_{p}, \rho_{j}\right)-\sum_{p>j} \ln X_{j, p}\left(\rho_{j}, \rho_{p}\right)\right) .
$$

The solution of this is

$$
S=\exp \left\{\frac{2 h \imath}{|\beta|^{2} \hbar} \sum_{b>a} \sum_{n=0}^{h-1} w^{n}\left(\gamma_{a}\right) \cdot\left(\gamma_{b}\right) L i_{2}\left[\exp \left(\rho_{a}-\rho_{b}+\frac{\imath \pi}{h}\left(\delta_{i_{b}, B}-\delta_{i_{a}, B}-2 n\right)\right)\right]\right\} .
$$

We have used the definition of the classical Euler dilogarithm

$$
L i_{2}(v)=\sum_{s=1}^{\infty} \frac{v^{s}}{s^{2}}=-\int_{0}^{v} \frac{\ln (1-y)}{y} d y .
$$

Many of the interesting properties of this function were studied by one William Spence in the early nineteenth century [20. A more recent discussion in the mathematical literature can be found in ref. [21]. Recent developments relating to conformal field theory are reviewed in ref. [22]. The matrix (3.4) satisfies various conditions, which are essentially related to properties of the time-delay functions $X_{i j}$. This function was studied recently in ref. [23, where it was noted that it 
has properties reminiscent of those of $S$ matrices. Indeed, one can think of these properties as being consequences of the fact that the $S$ matrix (3.4) is periodic, symmetric, etc. Let us define

$$
T_{a b}(\rho)=\sum_{n=0}^{h-1} w^{n}\left(\gamma_{a}\right) \cdot\left(\gamma_{b}\right) L i_{2}\left[\exp \left(\rho+\frac{\imath \pi}{h}\left(\frac{c(a)-c(b)}{2}-2 n\right)\right)\right] .
$$

Then the matrix function $T_{a b}(\rho)$ satisfies the following relations:

(i) $T_{a b}(\rho+21 \pi)=T_{a b}(\rho)$

(ii) $T_{a b}(\rho)=T_{b a}(\rho)$

(iii) $T_{a b}(\rho+i \pi)=-T_{\bar{a} b}(\rho)$

(iv) $\left(T_{a b}\left(\rho^{*}\right)\right)^{*}=T_{a b}(\rho)$

(v) $T_{a b}(\rho)=-T_{a b}(-\rho)+2 T_{a b}(0)$

(vi) $\sum_{t=i, j, k} T_{l t}\left(\rho+1 \eta_{t}\right)=0$, where $l$ is a free label and $i, j, k$ satisfy a fusing rule; $\eta_{t}=-2 \xi_{t}+\frac{c(t)-1}{2}$, where $c(t)$ is $1(-1)$ if the root $\alpha_{t}$ is black(white), and $\xi_{t}$ are the integers in the fusing relation $\sum_{t=i, j, k} \omega^{-\xi_{t}} \gamma_{t}=0$.

These relations can be shown directly: Property (i) is obvious; properties (ii), (iii) and (iv) follow using arguments analogous to those used for the time-delay functions $X_{a b}(\rho)$ in ref. [23]. Property (vi) similarly follows from an argument analogous to that given in ref. [7] when discussing the affine Toda particle $S$-matrix. Property (v) is most easily proved by noting firstly that the $\rho$ derivative of this equation is true - this follows using eqn. (3.5) and the fact that $X_{a b}(\rho)=X_{a b}(-\rho)$ (see ref. [23]). Thus the left-hand side of (v) equals the right-hand side up to an additive constant, and putting $\rho=0$ one sees that this constant must vanish. A direct proof of (v) seems more involved - for example, for the $A_{1}$ case this property reduces to the equation

$$
L i_{2}\left(-e^{\rho}\right)-L i_{2}\left(e^{\rho}\right)=-L i_{2}\left(-e^{-\rho}\right)+L i_{2}\left(e^{-\rho}\right)+2 L i_{2}(-1)-2 L i_{2}(1) .
$$

The Euler dilogarithm satisfies the following 'inversion' relation [22]

$$
L i_{2}(-y)+L i_{2}(-1 / y)=-\frac{\pi^{2}}{6}-\frac{1}{2}(\log y)^{2} .
$$

The function $L_{2}(y)$ is divergent for real $y, y>1$; however, one can define a continuous function on the real line by setting [22]

$$
L i_{2}(y)=\frac{\pi^{2}}{3}-L i_{2}(1 / y)-\frac{1}{2}(\log y)^{2}, \quad \text { for } y>1 \text {. }
$$


Then, using the above two equations and the facts that $L i_{2}(1)=\pi^{2} / 6$ and $L i_{2}(-1)=$ $-\pi^{2} / 12$, equation (3.7) may be proved.

The constant term in the relation (v) means that the $\mathrm{S}$ matrix (3.4) will be invariant under $\rho \rightarrow-\rho$ only if it is normalised so that $S(\rho=0)=1$. Physically this requirement is obvious - that there be no scattering when the two solitons have the same rapidity. We note that the properties (i)-(vi) above reflect identities satisfied by the Euler dilogarithm.

Thus the expression (3.4) satisfies relations expected for an $S$-matrix. Note, however, that the Euler dilogarithm can be continued to a multi-valued function on the complex plane, minus the segment $(1, \infty)$ of the real axis [21, 22]. Hence our proposed $S$-matrix does not have the expected pole structure. We will comment upon this in the following section.

\section{Conclusions and Developments}

It is important to be clear about what this S-matrix is, and what it is not. We have only made a semi-classical approximation to the quantum theory of affine Toda solitons, and so do not expect to see all of the behaviour typical of a quantum field theory. In particular as noted above, there are no poles in formula (3.4), and we have made no mention of the renormalisation of the soliton masses. The interested reader should consult the recent papers [24] and 25] for a discussion of this latter point. Very little is known about the expected behaviour of the poles on account of difficulties concerning the unitarity of the affine Toda theories in the imaginary coupling régime. Another feature expected of the quantum theory which this kind of approximation will not possess is that there will be no processes which change the topological charges of the scattering solitons. This is because such these are absent classically. An obvious conjecture worthy of exploration is that a full quantum version of (3.4) involves the quantum dilogarithm of ref. [26].

What we believe we have is the S-matrix of an quantum integrable particle theory, generalising that of Ruijsenaars [27, 28]. In the case of $\hat{A}$-series solitons of equal mass this particle model already reproduces the appropriate scattering shifts. In the more general case however, no such model is known. Starting from the formula for the shifts which follows from Ruijsenaars model, and fixing the rapidities in such a way that the soliton fusing rule [17] is satisfied, we have obtained correct expressions for the shifts when two solitons of different masses scatter (it is

reasonably easy to show that all of the $\hat{A}$-series solitons can be obtained by repeated fusing from the lightest in this manner). The question is whether this procedure can be implemented at the level of the Ruijsenaars Hamiltonian. This is a nontrivial problem since the fusings correspond to imposing imaginary constraints on 
the particle rapidities, and it is unclear what effect this will have on the symplectic structure of the phase space. We remark that the change $\rho \rightarrow \rho+2 i n \pi / h$ is a symplectic transformation, albeit a complex one, which gives us some hope that the above procedure can be made to work.

A natural generalisation of this work would seem to be to try and include the breathers. In the sine-Gordon theory at least these breathers are related to the particles, and possess a number of discrete energy levels according to the value of the coupling constant $\beta$. It is a long-standing problem in affine Toda soliton theory to try and characterise the breather spectrum for more general theories, and in particular to see if the particle-soliton correspondence remains. We have obtained preliminary results in this direction, including a description of the energy levels of

the $\hat{A}$-series breathers of remarkable and rather mysterious simplicity. We plan to discuss these issues in a forthcoming paper [29].

\section{Acknowledgements}

Part of this work was done under the auspices of a Royal Society Visiting Fellowship award to one of us (JWRU). JWRU thanks the late UK Science and Engineering Research Council, and BS acknowledges support from the Australian Research Council and the UK Engineering and Physical Sciences Research Council.

\section{References}

[1] A. Fring, H.C. Liao, and D.I. Olive. The mass spectrum and coupling in affine Toda field theory. Phys. Lett., B266:82, 1991.

[2] P.E. Dorey. Root systems and purely elastic S-matrices 2. Nucl. Phys., B374:741, 1992.

[3] P.E. Dorey. Root systems and purely elastic S-matrices. Nucl. Phys., B358:654, 1991.

[4] H.W. Braden, E. Corrigan, P. E. Dorey, and R. Sasaki. Extended Toda field theory and exact S-matrices. Phys. Lett., B227:411, 1989.

[5] H.W. Braden, E. Corrigan, P. E. Dorey, and R. Sasaki. Talk. In Proc. XVIII Int. Conf. on Differential Geometric Methods in Theoretical Physics: Physics and Geometry, Lake Tahoe., 1989.

[6] H.W. Braden, E. Corrigan, P. E. Dorey, and R. Sasaki. Affine Toda field theory and exact S-Matrices. Nucl. Phys., B338:465, 1990.

[7] A. Fring and D.I. Olive. The fusing rule and scattering matrix of affine Toda theory. Nucl. Phys., 379B:429, 1992. 
[8] T.R. Klassen and E. Melzer. Purely elastic scattering theories and their ultraviolet limits. Nucl. Phys., B338:485, 1990.

[9] E. Corrigan and P.E. Dorey. A representation of the exchange relation for affine Toda field theory. Phys. Lett., B273:237, 1991.

[10] M. Freeman. On the Mass Spectrum of Affine Toda Field Theory. Phys. Lett. B261:57, 1991.

[11] T.J. Hollowood. Solitons in affine Toda field theories. Nucl. Phys., B384:523, 1992.

[12] H. Aratyn et. al. Hirota's solitons in the affine and conformal affine Toda model. Nucl. Phys. B406:727, 1993.

[13] D.G. Caldi and Z. Zhu. Multisoliton solutions of affine Toda models. SUNY Buffalo preprint UB-TH-0193, hep-th/9307179.

[14] A.N. Leznov and M.V. Saveliev. Group-Theoretical Methods for Integration of Nonlinear Dynamical Systems, volume 15 of Progress in Physics. BirkhauserVerlag, Basel, 1992.

[15] D.I. Olive, N. Turok, and J.W.R. Underwood. Solitons and the energymomentum tensor for affine Toda theory. Nucl. Phys., B401:663, 1993.

[16] J.W.R. Underwood. Aspects of non-abelian Toda theories. Preprint IC/TP/92-93/30, 1993.

[17] D.I. Olive, N. Turok, and J.W.R. Underwood. Affine Toda solitons and vertex operators. Nucl. Phys., B409:509, 1993.

[18] G. Papadopoulos and B. Spence. The space of solutions of Toda field theory. Mod. Phys. Lett, A9:2469, 1994.

[19] M.A.C. Kneipp and D.I. Olive. Crossing and antisolitons in affine Toda theories. Nucl. Phys., B408:565, 1993.

[20] W. Spence. An essay on logarithmic transcendents, London and Edinburgh 1809.

[21] L. Lewin. Polylogarithms and Associated Functions. Elsevier-North Holland, 1981.

[22] A. Kirillov. Dilogarithm Identities. Univ. Tokyo/Steklov Institute preprint, hepth/9407047. 
[23] A. Fring, P.R. Johnson, M.A.C. Kneipp and D.I. Olive. Vertex Operators and Soliton Time Delays in Affine Toda Field Theory. Swansea preprint SWAT/9394/30, hep-th/9405034.

[24] N.J. MacKay and G.M.T. Watts. Quantum mass corrections for affine toda solitons. Preprint DAMTP 94/96, SPhT T94/137, hep-th/9411169, 1994.

[25] G. Delius and M. Grisaru. Toda Soliton Mass Corrections and the ParticleSoliton Duality Conjecture. Kings College London/Brandeis preprint, KCLTH-94-18, BRX-TH-362, hep-th/941117.

[26] L.D. Faddeev and R.M. Kashaev. Quantum Dilogarithm. Mod. Phys. Lett. A9:427, 1994.

[27] S.N.M. Ruijsenaars and H. Schneider. A New Class of Integrable Systems and Its Relation to Solitons. Ann. Phys. 170:370, 1986.

[28] S.N.M. Ruijsenaars. Complete Integrability of Relativistic Calogero-Moser Systems and Elliptic Function Identities. Commun. Math. Phys. 110:191, 1987.

[29] W. Spence and J.W.R. Underwood. in preparation. 